\title{
Salvageability of renal function following renal revascularisation in children with Takayasu's arteritis-induced renal artery stenosis
}

\author{
P N Obiagwu, ${ }^{1,2}$ MBBS, FWACP (Paeds); P Gajjar, ${ }^{2}$ MB ChB, FCPaed (SA); M McCulloch, ${ }^{3}$ MB BCh, FCPaed (SA), FRCPCH; \\ C Scott, ${ }^{4} \mathrm{MB}$ BCh, FCPaed (SA); A Numanoglu, ${ }^{5} \mathrm{MB}$ ChB, FCS (SA); P Nourse, ${ }^{2}$ MB ChB, FCPaed (SA) \\ ${ }^{1}$ Paediatric Nephrology Unit, Aminu Kano Teaching Hospital, Kano, Nigeria \\ ${ }^{2}$ Paediatric Nephrology Unit, Red Cross War Memorial Children's Hospital, Cape Town, South Africa \\ ${ }^{3}$ Paediatric Intensive Care Unit, Red Cross War Memorial Children's Hospital, Cape Town, South Africa \\ ${ }^{4}$ Paediatric Rheumatology Unit, Red Cross War Memorial Children's Hospital, Cape Town, South Africa \\ ${ }^{5}$ Paediatric Surgery Unit, Red Cross War Memorial Children's Hospital, Cape Town, South Africa
}

Corresponding author: P N Obiagwu (patience.obiagwu@gmail.com)

\begin{abstract}
Background. Renal artery revascularisation procedures are usually carried out on children with renal artery stenosis from varied causes, including Takayasu's arteritis. Reports on the outcome of such procedures in children usually refer to the improvement in blood pressure, with only minimal mention of effects on renal function.

Objective. Salvageability of renal function in children who underwent renal revascularisation for Takayasu's arteritis-induced renal artery stenosis (TARAS) was the focus of this study.

Methods. We undertook a retrospective analysis of children aged $\leq 16$ years with angiographically confirmed TARAS who underwent renal artery revascularisation procedures between 1990 and 2010. Outcomes of renal function were studied over a period of 2 years and were defined as: (i) improvement: $>20 \%$ increase in estimated glomerular filtration rate (e-GFR) from presurgery value; (ii) stabilisation: e-GFR within $20 \%$ of presurgery value; and (iii) failure: $>20 \%$ deterioration in e-GFR from presurgery value. The GFR was estimated using the Schwartz formula.

Results. Twenty children (9 males and 11 females, age range 2 - 14 years) had 27 renal artery revascularisation procedures. Thirteen of the patients (65.0\%) had bilateral renal artery stenosis. The baseline mean e-GFR was 88.6 (standard deviation (SD) 25.4 ) $\mathrm{mL} / \mathrm{min} / 1.73 \mathrm{~m}$ and the mean duration of follow-up was 28.80 (SD 25.62) months. All the patients had stable or improved renal function until the 2-year follow-up, when the proportion decreased to $92.3 \%$ (12/13), as failure was recorded in one child. Bilateral revascularisation was found to be significantly associated with improvement in renal function in the early postoperative period $(p=0.04)$.

Conclusion. Renal artery revascularisation procedures are successful in salvaging renal function in children with TARAS.
\end{abstract}

S Afr Med J 2016;106(8):813-816. DOI:10.7196/SAMJ.2016.v106i8.10490

Takayasu's arteritis is a rare, chronic inflammatory and stenotic disease of medium- and large-sized arteries, characterised by a predilection for the aortic arch and its branches. ${ }^{[1,2]}$ It is predominantly a disease of young adults in the second and third decades of life. However, its onset can be earlier in childhood, ${ }^{[3]}$ and cases have been described in infants. ${ }^{[4-6]}$ The inflammatory nature of the disease results in a wide range of vascular lesions including varying degrees of arterial stenosis, occlusion or aneurysmal dilation of the involved vessels. Stenosis often results in visceral ischaemia, and the disease frequently affects the kidney. ${ }^{[7,8]}$

Involvement of the renal arteries is fairly common in children ${ }^{[1,7-9]}$ and is associated with severe hypertension and chronic kidney disease, with consequent significant morbidity and mortality. In previous studies from South Africa (SA), the renal arteries were affected in $78-86 \%$ of cases. ${ }^{[7,9]}$ Kidney revascularisation procedures are often required to control hypertension or to salvage renal function.

Reports on renal function outcomes of revascularisation procedures in children and adults are scarce and poorly reported. Differences in the disease pattern between children and adults, ${ }^{[10]}$ as well as the fact that children are still growing, may affect the outcome of interventions, necessitating studies in children. We recently reported on the outcome of hypertension in a cohort of patients with Takayasu's arteritis, but did not adequately report on their renal function outcomes. ${ }^{[1]]}$ Owing to the progressive nature of the disease, renal function may continue to deteriorate despite adequate blood pressure control.

\section{Objective}

To determine the salvageability of renal function following revascularisation procedures in the same cohort of children with Takayasu's arteritis-induced renal artery stenosis (TARAS) in our centre.

\section{Methods}

This was a 21-year retrospective review of the database of all children with TARAS who had renal artery revascularisation procedures between 1 January 1990 and 31 December 2010 at Red Cross War Memorial Children's Hospital, Cape Town, SA. The hospital is a tertiary referral centre that provides specialist care to all children from referral regions of the Western Cape and Eastern Cape provinces. Diagnosis of Takayasu's arteritis was based on the European League Against Rheumatism/Paediatric Rheumatology European Society (EULAR/PReS) endorsed consensus criteria for the classification of childhood vasculitides, ${ }^{[12]}$ and on the Ishikawa criteria ${ }^{[13]}$ before the year 2006. Renal artery stenosis, usually determined using Doppler ultrasonography and computed tomography angiography, is defined 
as stenosis of the main renal artery or its proximal branches and is considered significant if $>50 \%$ of the lumen is involved.

Children diagnosed with Takayasu's arteritis are managed by a multidisciplinary team comprising the paediatric nephrology, rheumatology and surgical units. All children were initially managed based on the hospital's standard medical management protocol, which includes: (i) antituberculosis treatment for 6 months with three drugs (as there is an extremely high prevalence of tuberculosis in the hospital drainage area, associated with a positive tuberculin skin test in $>90 \%$ of our patients with Takayasu's arteritis $\left.{ }^{[11]}\right)$; (ii) immunosuppression with pulse methylprednisolone (followed by daily oral prednisone, which is gradually tailed off over 2 3 months), a single dose of intravenous cyclophosphamide at $500-750 \mathrm{mg} / \mathrm{m}^{2}$, and oral methotrexate at $10-15 \mathrm{mg} / \mathrm{m}^{2} /$ week; and (iii) antihypertensives as indicated.

Following acute reduction in disease activity, as determined by the absence of signs and symptoms of systemic disease such as fever and malaise, as well as normalisation of the erythrocyte sedimentation rate (ESR), surgical procedures are performed for children with refractory hypertension (unresolved hypertension after 3 months on at least three antihypertensive drugs from different classes, including a diuretic $\left.^{[14]}\right)$, severe side-effects of medication, medication intolerance and/or deterioration in renal function as determined by serum creatinine ( $\mathrm{sCr}$ ) and glomerular filtration rate (GFR) estimations and aneurysms. The surgical procedures performed include percutaneous transluminal angioplasty (PTA), kidney autotransplantation, arterial bypass surgery using either autologous or prosthetic vascular grafts, or revascularisations using splenic, hepatic and other arteries. Some children also had a nephrectomy.

Criteria for success were adopted from the American Heart Association guidelines for reporting of renal artery revascularisation in clinical trials. ${ }^{[15]}$ Renal function was monitored using serial $\mathrm{sCr}$ levels and estimated glomerular filtration rates (eGFRs). The GFR was estimated using the modified Schwartz formula (eGFR corrected for gender and age). ${ }^{[16]}$ The baseline $\mathrm{sCr}$ concentration was taken to be the level closest to the date of operation, all within 1 week of surgery. Subsequent $\mathrm{sCr}$ concentrations to document renal function response were recorded at 2 weeks - 3 months, 3 - 6 months, 6 - 12 months and $12-24$ months after surgery. The renal response to revascularisation was assessed as follows: (i) improvement: $>20 \%$ increase in e-GFR from presurgery value; (ii) stabilisation: e-GFR within $20 \%$ of presurgery value; and (iii) failure: $>20 \%$ deterioration in e-GFR from presurgery value.

\section{Ethical considerations}

As this was a review of a database, all patient records were anonymised and deidentified prior to analysis. This retrospective study was approved by the Human Research Ethics Committee of the Faculty of Health Sciences, University of Cape Town (HREC Ref.: 026/2013).

\section{Statistical analysis}

All data were analysed using SPSS 20.0 software (IBM, USA). Continuous data were presented as means (standard deviations (SDs)). Differences in continuous data were analysed with Student's $t$-test, while those for categorical data were analysed using the $\chi^{2}$ test. A $p$-value of $<0.05$ was considered to be statistically significant in all analyses.

\section{Results}

Fifty-nine children with Takayasu's arteritis were managed during the 21-year study period. Of these, 20 (34.0\%), with ages ranging between 2 and 14 years, had renal artery revascularisation for
TARAS. All of them were significantly hypertensive. Of the children who had revascularisation surgery, $13(65.0 \%)$ had bilateral renal artery stenosis. The mean duration of follow-up was 28.80 (SD 25.62) months (range 1 - 81). Demographic data and laboratory values for the children who had revascularisation surgery are shown in Table 1. No child was on renal replacement therapy.

\section{Renal function outcomes}

The 20 children underwent 27 revascularisation procedures. Six of the children had bilateral revascularisation for significant bilateral disease. Of these 6, 1 had another revascularisation procedure carried out on one side because of a previously unsuccessful procedure. The remaining 7 children who had bilateral disease had revascularisation procedures carried out on the side with the more significant stenosis. Four children had undergone nephrectomy before revascularisation, while 3 had nephrectomy done after the revascularisation surgery. All but 1 of the children had the revascularisation carried out in our facility. The commonest procedure was PTA, which was done singly or in combination with other procedures in 10 patients (50.0\%). The procedures done were as follows: (i) PTA: 10 patients; (ii) autotransplants: 9 patients; (iii) revascularisations using splenic and hepatic arteries: 6 patients; and (iv) arterial bypasses with autologous grafts: 2 patients.

Renal function outcomes were available for 17 (85.0\%), 17 (85.0\%), $14(70.0 \%)$ and $13(65.0 \%)$ patients at the 3-month, 6-month, 1 -year and 2-year follow-up periods, respectively. Five children had returned to the regions from which they had been referred -1 to another country and 4 to other regions within SA. Within the first 3 months after revascularisation, $8 / 17$ patients $(47.1 \%)$ experienced

Table 1. Demographic and laboratory variables for children who underwent revascularisation surgery for TARAS $(N=20)$

\begin{tabular}{ll}
\hline Variable & Value \\
\hline $\begin{array}{l}\text { Age (years), mean (SD) } \\
\text { Gender, } n(\%)\end{array}$ & $9.1(3.1)$ \\
$\quad$ Males & $9(45.0)$ \\
$\quad$ Females & $11(55.0)$ \\
Hypertension, $n(\%)$ & $20(100.0)$ \\
Baseline ESR $(\mathrm{mm} / \mathrm{h})$, range (median) & $5-143(52.9)$ \\
Baseline sCr $(\mu \mathrm{mol} / \mathrm{L})$, range, mean $(\mathrm{SD})$ & $53-150,88.6(25.4)$ \\
Side of renal artery stenosis, $n(\%)$ & \\
$\quad$ Right & $5(25.0)$ \\
$\quad$ Left & $2(10.0)$ \\
$\quad$ Bilateral & $13(65.0)$
\end{tabular}

Table 2. Mean eGFR values at different follow-up periods

\begin{tabular}{|c|c|c|}
\hline Period under review & Patients, $n$ & $\begin{array}{l}\text { eGFR values }(\mathrm{mL} / \\
\left.\mathrm{min} / 1.73 \mathrm{~m}^{2}\right) \text {, mean }(\mathrm{SD})\end{array}$ \\
\hline Before revascularisation & 20 & $88.6(25.4)$ \\
\hline \multicolumn{3}{|l|}{ After revascularisation } \\
\hline 0 - 3 months & 17 & $101.4(24.7)$ \\
\hline 3 - 6 months & 17 & $98.3(19.5)$ \\
\hline $6-12$ months & 14 & $98.8(24.5)$ \\
\hline $12-24$ months & 13 & $95.2(22.7)$ \\
\hline
\end{tabular}


an improvement in their renal function, with 9 (52.9\%) remaining stable. By 6 months, 9/17 patients (52.9\%) had improved renal function, the renal function of the remaining 8 patients (47.1\%) remaining essentially unchanged. Subsequent evaluations at 1 and 2 years after revascularisation showed improvement in 6/14 (42.9\%) and $5 / 13(38.5 \%)$ patients, respectively, while deterioration in renal function was recorded in 1 patient $(7.7 \%)$ at the 2 -year follow up. All the other children had stable renal function. Ninety-two percent of the patients therefore had stable or improved renal function at longterm follow-up $(p<0.05)$.

The mean (SD) eGFR values at the various follow-up periods are shown in Table 2. There was no significant association between age, sex, type of revascularisation procedure, number of antihypertensive medications or blood pressure control and renal outcome of revascularisation at the various follow-up periods ( $p>0.05$ at each period). However, bilateral revascularisation was found to be significantly associated with an improvement in renal function in the early 3 -month postoperative period $(p=0.04)$. This significance was not observed at further longer-term follow-up periods.

\section{Discussion}

Revascularisation procedures have increasingly been used in the management of renal artery stenosis refractory to medical management. There are few reports on the efficacy of renal artery revascularisation in salvaging renal function in children with renal artery stenosis from various causes, including Takayasu's arteritis. ${ }^{[17-23]}$ In fact, before the report by Piercy et al. ${ }^{[19]}$ there were no articles reporting on changes in estimated GFR, as is the currently accepted norm..$^{[15,20-22]}$ Prior to this, renal function was often reported as the mean of absolute $\mathrm{sCr}$ values or as a function of the number of patients with chronic kidney disease. This gives no indication of the success or failure of the intervention in salvaging renal function.

There is in fact only one report on children with Takayasu's arteritis that reported on renal function after revascularisation. ${ }^{[21]}$ In 33 children, Feng et al. ${ }^{[21]}$ demonstrated an improvement in e-GFR in $36 \%$, stabilisation in $52 \%$ and failure in only $4 \%$. This was similar to our group of children with TARAS, almost all of whom had improved or stable renal function on follow-up, with only one child exhibiting deterioration in renal function on long-term followup. Other studies from the USA ${ }^{[19,24]}$ and the $\mathrm{UK}^{[20,25]}$ have shown similar findings of salvageable renal function following surgical procedures in patients with renal artery stenosis, with much higher rates of success but mainly in diseases other than Takayasu's arteritis. In adults with Takayasu's arteritis, as reported by Weaver et al., ${ }^{[26]}$ renal revascularisation resulted in the improvement or stabilisation of renal function in almost all the patients studied, including reversing the dialysis dependence of 2 of the 3 patients who were dialysis dependent preoperatively. Similar findings were reported by Ham et al., ${ }^{[27]}$ who studied adults with non-atherosclerotic renal artery disease and found significant improvement in renal function, especially in those with Takayasu's arteritis.

Monitoring and reporting on renal function is vital, and small changes in GFR could alert the practitioner to the possibility of progressive disease that may warrant further imaging and possible intervention. A child could have deteriorating renal function while his or her blood pressure is controlled on antihypertensive medications. Children with Takayasu's arteritis, as opposed to adults, often have active disease that may progress. Patients with active disease have been shown in several studies to have worse outcomes after surgical intervention. ${ }^{[21,28,29]}$ It is therefore important to monitor their renal function regularly to detect any deterioration. In their series, Stanley et al. ${ }^{[24]}$ reported no case of potential worsening of renal function despite the loss of renal mass associated with nephrectomy, which was carried out in a number of their cases. This is similar to our findings in the cases in which nephrectomy was done. It probably emphasises the known fact that a well-perfused kidney will compensate for lack of function on the other side, and further stresses the importance of monitoring for small changes in GFR. In our centre, we have started performing nuclear medicine studies with individualised GFR measurements to monitor the GFR of individual kidneys. This is because absolute creatinine values alone with overall e-GFRs are probably not sensitive enough to detect loss of function in individual kidneys.

The commonest procedure performed was PTA, which was done in half of the patients, either singly or in combination with other revascularisation procedures. Studies have shown good success both technically and clinically using PTA in children with Takayasu's arteritis, ${ }^{[1,30-32]}$ as well as in those without. ${ }^{[18,22,23,33,34]}$ However, only Chalmers et al. ${ }^{[18]}$ and Huang et al. ${ }^{[22]}$ reported on renal function, and with good outcomes. One of the complications reported in the studies in which PTA was carried out is restenosis, the rates of which vary between different reports. While the underlying diagnosis may well play a role in this complication, the site and nature of the stenosis, the techniques used and the decision whether or not to insert a stent vary in these reports, making comparison of success rates difficult. Surgical revascularisation has been shown to have a better outcome than PTA in adult patients with Takayasu's arteritis in terms of patency rates and postoperative complications. ${ }^{[27,35,36]}$ A recent paediatric report from the USA which included patients with Takayasu's arteritis also showed a higher rate of reintervention with PTA compared with surgery. ${ }^{[23]}$ Despite the slightly higher complication rate, many authors would still recommend PTA as the first choice where possible in children. ${ }^{[20,30,32]}$ This is because PTA may be possible when children are too small to be operated on. PTA is a simple, cost-effective procedure, and is an attractive option in developing countries where vascular surgeons may not be available. Advancing endovascular techniques such as cutting wires, highpressure balloons and different types of stents may further contribute to the increasing use of PTA in the future. ${ }^{[23,33]}$

Two of our patients had an arterial bypass using the saphenous vein. Although there have been reports of aneurysmal dilatation with this approach, ${ }^{[24]}$ a recent report from China showed a very good outcome in children with Takayasu's arteritis-induced renovascular hypertension in terms of hypertension and function. ${ }^{[21]}$ Revascularisation using other arteries, such as spleno-renal, gastroduodenal-renal, hepato-renal and ilio-renal bypass surgery, has been documented to have a high restenosis rate. In our series, although the bypass surgeries were not successful in controlling hypertension, ${ }^{[11]}$ we were able to preserve renal function.

\section{Study limitations}

This study was a retrospective review of data spanning over two decades. This proved to be a limitation, as during this period there was considerable evolution of management protocols, both medical and surgical, as well as advances in interventional techniques and technology. In addition, the diagnostic criteria for the classification of childhood vasculitides have evolved over time, and have been adopted by EULAR/PReS. ${ }^{[12]}$ These criteria are nonspecific, and it is possible that a few patients with other diagnoses may have been included in our study.

\section{Conclusion}

Renal revascularisation in children with Takayasu's arteritis can yield a significant benefit in long-term renal function. It may be impor- 
tant to consider the procedure before the patient develops significant renal dysfunction, after which this benefit may be much reduced. The retrospective nature of our study, which invariably implies lost data, the lack of serial nuclear GFRs of the patients, and the small number of patients studied could be limitations.

Acknowledgements. The authors wish to acknowledge the International Society of Nephrology and the International Paediatric Nephrology Association for sponsoring the corresponding author (PNO) in paediatric nephrology training at Red Cross War Memorial Children's Hospital, Cape Town

\section{References}

1. Jennette JC, Falk RJ, Bacon PA, et al. 2012 revised International Chapel Hill consensus conference nomenclature of vasculitides. Arthritis Rheum 2013;65(1):1-11. DOI:10.1002/art.37715

2. Gulati A, Bagga A. Large vessel vasculitis. Pediatr Nephrol 2010;25(6):1037-1048. DOI:10.1007/ s00467-009-1312-9.

3. Ladhani $\mathrm{S}$, Tulloh $\mathrm{R}$, Anderson D. Takayasu disease masquerading as an interruption of the aortic arch in a 2 year old child. Cardiol Young 2001;11(2):244-246. DOI:10.1017/S104795110100021X

4. Eke F, Balfe JW, Hardy BE. Three patients with arteritis. Arch Dis Child 1984;59(9):877-883. DOI:10.1136/adc.59.9.877.

5. Mitchell CS, Parisi MT. Magnetic resonance imaging of Takayasu's aortitis in an infant. J Am Osteopath Assoc 1997;97(10):607-609.

6. Kierzkowska B, Lipinska J, Baranska D, et al. Takayasu's arteritis mimicking Kawasaki disease in 7-month-old infant, successfully treated with glucocorticosteroids and intravenous immunoglobulins. Rheumatol Int 2012;32(11):3655-3659. DOI:10.1007/s00296-010-1518-y

7. McCulloch M, Andronikou S, Goddard E, et al. Angiographic features of 26 children with Takayasu's arteritis. Pediatr Radiol 2003;33(4):230-235. DOI:10.1007/s00247-002-0817-1

8. Cakar N, Yalcinkaya F, Duzova A, et al. Takayasu arteritis in children. J Rheumatol 2008;35(5):913-919. 9. Hahn D, Thomson PD, Kala U, Beale PG, Levin SE. A review of Takayasu's arteritis in children in Gauteng, South Africa. Pediatr Nephrol 1998;12(8):668-675. DOI:10.1007/s004670050526

10. Brunner J, Feldman BM, Tyrrell PN, et al. Takayasu arteritis in children and adolescents. Rheumatology (Oxford) 2010;49(10):1806-1814. DOI:10.1093/rheumatology/keq167

11. Ladapo TA, Gajjar P, McCulloch M, Scott C, Numanoglu A, Nourse P. Impact of revascularization on hypertension in children with Takayasu's arteritis-induced renal artery stenosis: A 21-year review. on hypertension in children with Takayasu's arteritis-induced renal artey st
Pediatr Nephrol 2015;30(8):1289-1295. DOI:10.1007/s00467-015-3049-y

12. Ozen S, Ruperto N, Dillon MI, et al. EULAR/PReS endorsed consensus criteria for the classification of Ozen S, Ruperto N, Dillon MJ, et al. EULAR/PReS endorsed consensus criteria for the classificat
childhood vasculitides. Ann Rheum Dis 2006;65(7):936-941. DOI:10.1136/ard.2005.046300

13. Ishikawa K. Natural history and classification of occlusive thromboaortopathy (Takayasu's disease) Circulation 1978:57(1):27-35. DOI:10.1161/01.CIR.57.1.27

14. National Kidney Foundation. Kidney Disease Outcome Quality Initiative. Clinical Practice Guidelines on Hypertension and Antihypertensive Agents in Chronic Kidney Disease. Guideline 4: Evaluation fo renal artery disease. Am J Kidney Dis 2004;43(S1):11-13. DOI:10.1053/j.ajkd.2004.03.003

15. Rundback JH, Sacks D, Kent KC, et al. Guidelines for the reporting of renal artery revascularization in clinical trials. Circulation 2002;106(12):1572-1585. DOI:10.1161/01.CIR.0000029805.87199.45

16. Schwartz GJ, Munoz A, Schneider MF, et al. New equations to estimate GFR in children with CKD. Am Soc Nephrol 2009:20(3):629-637. DOI:10.1681/ASN.200803028
17. Valdes F, Saieh C, Kramer A. Surgical treatment of complete renal artery occlusion in pediatric patients. Ann Vasc Surg 1990;4(5):490-493. DOI:10.1016/S0890-5096(07)60076-4

8. Chalmers RT, Dhadwal A, Deal JE, Sever PS, Wolfe JH. The surgical management of renovascular hypertension in children and young adults. Eur I Vasc Endovasc Surg 2000;19(4):400-405. DOI:10.1053/ejvs.1999.1020

19. Piercy KT, Hundley JC, Stafford JM, et al. Renovascular disease in children and adolescents. J Vasc Surg 2005;41(6):973-982. DOI:10.1016/j.jvs.2005.03.007

20. Stadermann MB, Montini G, Hamilton G, et al. Results of surgical treatment for renovascular hypertension in children: 30-year single centre experience. Nephrol Dial Transplant 2010;25(3):807813. DOI:10.1093/ndt/gfp537

21. Feng R, Wei $\mathrm{X}$, Zhao Z, et al. Aortorenal bypass with autologous saphenous vein in Takayasu arteritis-induced renal artery stenosis. Eur J Vasc Endovasc Surg 2011;42(1):47-53. DOI:10.1016/j. ejvs.2011.03.004

22. Huang Y,Duncan AA, McKusick MA, et al. Renal artery intervention in pediatric and adolescent patients: A 20-year experience. Vasc Endovasc Surg 2008;41(6):490-499. DOI:10.1177/1538574407307944

23. Porras D, Stein DR, Ferguson MA, et al. Midaortic syndrome: 30 years of experience with endovascular and surgical management. Pediatr Nephrol 2013;28(10):2023-2033. DOI:10.1007/s00467-013-2514-8

24. Stanley JC, Criado E, Upchurch GR Jr, et al. Pediatric renovascular hypertension: 132 primary and 30 Stanley JC, Criado E, Upchurch GR Jr, et al. Pediatric renovascular hypertension: 132 primary and 30
secondary operations in 97 children. J Vasc Surg 2006;44(6):1219-1228. DOI:10.1016/j.jvs.2006.08.009

25. Boondary operations in 97 children. J Vasc Surg 2006;44(6):1219-1228. DOI:10.1016/j.jvs.2006.08.009 1 and the role of percutaneous transluminal angioplasty. Nephrol Dial Transplant 2002;17(7):12351240. DOI:10.1093/ndt/17.7.1235

26. Weaver FA, Kumar SR, Yellin AE, et al. Renal revascularization in Takayasu arteritis-induced renal artery stenosis. J Vasc Surg 2004;39(4):749-757. DOI:10.1016/j.jvs.2003.12.022

27. Ham SW, Kumar SR, Wang BR, Rowe VL, Weaver FA. Late outcomes of endovascular and open revascularization for nonatherosclerotic renal artery disease. Arch Surg 2010;145(9):832-839. DOI:10.1001/archsurg. 2010.183

28. Sunamori M, Hatano R, Yamada T, Tsukuura T, Sakamoto T. Aortitis syndrome due to Takayasu's disease: A guideline for the surgical indication. J Cardiovasc Surg 1976;17(5):443-456.

29. Miyata T, Sato O, Koyama H, Shigematsu H, Tada Y. Long-term survival after surgical treatment of patients with Takayasu's arteritis. Circulation 2003:108(12):1474-1480. DOI:10.1161/01. CIR.0000089089.42153.5E

30. Numan F, Hasanefendioglu Bayrak A, Cantasdemir M, Ozer H, Gulsen F. Percutaneous transluminal angioplasty in pediatric patients with Takayasu arteritis: Comparison of initial and long-term results of interventions on aorta and non-aortic vessels. Anadolu Kardiyol Derg 2010;10(3):281-284. DOI:10.5152/akd.2010.071

31. Sharma S, Gupta H, Saxena A, et al. Results of renal angioplasty in nonspecific aortoarteritis (Takayasu disease). J Vasc Interv Radiol 1998;9(3):429-435. DOI:10.1016/S1051-0443(98)70294-5

32. Tyagi S, Kaul UA, Satsangi DK, Arora R. Percutaneous transluminal angioplasty for renovascular hypertension in children: Initial and long-term results. Pediatrics 1997;99(1):44-49. DOI:10.1542/ peds.99.1.44

33. Shroff R, Roebuck DJ, Gordon I, et al. Angioplasty for renovascular hypertension in children: 20-year experience. Pediatrics 2006;118(1):268-275. DOI:10.1542/peds.2005-2642

34. Srinivasan A, Krishnamurthy G, Fontalvo-Herazo L, et al. Angioplasty for renal artery stenosis in pediatric patients: An 11-year retrospective experience. J Vasc Interv Radiol 2010;21(11):1672-1680. DOI:10.1016/j.jvir.2010.07.012

35. Saadoun D, Lambert M, Mirault $T$, et al. Retrospective analysis of surgery versus endovascular intervention in Takayasu arteritis: A multicentre experience. Circulation 2012;125(6):813-819. DOI:10.1161/CIRCULATIONAHA.111.058032

36. Cong XL, Dai SM, Feng X, et al. Takayasu's arteritis: Clinical features and outcomes of 125 patients in China. Clin Rheumatol 2010;29(9):973-981. DOI:10.1007/s10067-010-1496-1

Accepted 14 January 2016 